\title{
Rectangular Microstrip Antenna Using Inductive Septums for Dual Band Operation with a New Resonant Mode
}

\author{
Rajeev Ranjan, Manish Kr. Verma, Shounak Mukherjee, Dhrubajyoti Ghosh, Sudipta Chattopadhyay
}

Department of ECE, Siliguri Institute of Technology, Siliguri, Darjeeling, India.

Email: piyalirekha@yahoo.com

Received April 14 $4^{\text {th }}, 2012$; revised May $18^{\text {th }}, 2012$; accepted June $7^{\text {th }}, 2012$

\begin{abstract}
A novel rectangular microstrip antenna on conventional dielectric substrate with centrally loaded inductive septums are proposed for dual band operation. The proposed antenna has been thoroughly investigated and the results are presented in the paper. The conventional patch antenna fabricated on common substrates always resonates at its dominant $\mathrm{TM}_{10}$ mode which produces the radiation field along its broadside direction. In the present investigation, the same microstrip antenna is designed on conventional substrate with centrally located inductive septums beneath the patch, with a view to develop a new resonant mode which will produce radiation like dominant $\mathrm{TM}_{10}$ mode. The speciality of the proposed antenna is to excite a new resonant mode with good impedance bandwidth while the traditional dominant $\mathrm{TM}_{10}$ mode is kept unaltered. A thorough quantitative analysis is presented to justify the reason of generation of new resonant mode along with the traditional dominant $\mathrm{TM}_{10}$ mode. An easy and handful formulation has been established for calculation of the frequency for new resonant mode as a function of antenna parameters and the gap between the septums. The proposed idea has been verified through a commercial software package for a patch operating in $\mathrm{C}$ band and an excellent agreement is revealed.
\end{abstract}

Keywords: Dual Band; Inductive Septums; New Resonant Mode; Traditional $\mathrm{TM}_{10}$ Mode

\section{Introduction}

The rectangular microstrip antenna is the most common and popular antenna for its well known striking features, such as low profile, light weight, and compatibility with monolithic microwave integrated circuits and thus it finds growing number of new applications day by day [1]. In the modem era of communication technology, these antennas are very useful for satellite links (GPS, vehicular, etc.), as well as emerging applications, such as wireless local area networks (WLAN).

The conventional patch antenna fabricated on common substrates usually resonates at its dominant $\mathrm{TM}_{10}$ mode with a typical bandwidth of $2 \%-3 \%$. This is an intrinsic limitation in bandwidth, which is due to the resonant nature of the patch structure. Hence a new drive is given in research to overcome the bandwidth limitations of patch antennas. The applications, where the increased bandwidth is needed for operating at two separate subbands, a valid alternative to the broadening of total bandwidth is represented by dual-frequency patch antennas as indicated in [2]. Several efforts have been given to develop the dual frequency microstrip antennas and the most simple technique to realize it by exciting the two nearby orthogonal modes such as $\mathrm{TM}_{10}$ and $\mathrm{TM}_{01}$ is as reported in [3]. These antennas are characterized by two resonances with orthogonal polarizations and hence are known as orthogonal mode dual-frequency patch antennas. The inherent restriction of this approach is that the two different frequency bands excite two orthogonal polarizations. However, this method is very useful in lowcost short-range applications, where polarization requirements are not vital. Another approach to design such an orthogonal-mode dual band antenna using a single feed with a slot inclined with respect to the microstrip feed line is reported in [4]. Some more investigations such as $[5,6]$ have been reported which show that the dual band antennas can be designed with the help of multiple radiating elements in stacked configuration. The most popular technique for obtaining a dual-band operation is to introduce a reactive loading to a single patch such as stubs was first studied in [7]. A more practical configuration of [7] is presented in [8], in which the stub is constituted by a microstrip. A rectangular notch introduced at the radiating edge [9] is an alternative way to introduce a dual-frequency behavior. A rectangular patch loaded with two parallel slots close to the radiating edges and meandered with slits at the non-radiating edges for dual band operation is obtained from open literature 
[10]. A new design of single-feed, reduced-size dual-frequency rectangular microstrip antenna with a cross slot of equal length is presented in [11]. The characteristics of a single-feed dual-frequency compact microstrip antenna with a shorting pin are studied in [12]. One recently reported article [13], proposes an antenna which consists of a small circular patch surrounded by two concentric annular-rings, which is loaded by an unequal lateral cross-slot ground plane to produce dual band operation.

These various techniques to achieve dual-band operation from various types of microstrip antennas still suffers from either complex manufacturing process or the polarization purity in its radiation. Moreover, there are hardly some efficient design guidelines reported, which can be utilized successfully to design a microstrip antenna for dual band operation.

In order to alleviate the lacunae in those structures, a new technique is proposed where a pair of simple thin metal plate has been introduced beneath the patch centrally along the width of the patch as shown in Figure 1. This pair of simple thin metal plate produces an inductive discontinuity along the central region below the patch and hence acts as inductive septums. Unlike the previous structures we have introduced the reactive loading beneath the patch instead of giving it to the patch. This has been done with a view to generate a new resonant mode while the usual dominant $\mathrm{TM}_{10}$ mode is kept unaltered. The proposed structure has been conceived based on the conjecture of producing new effective radiating slots (responsible for generating new resonant mode) along with the actual radiating slots (responsible for generating traditional $\mathrm{TM}_{10}$ mode) by introducing the septums are justified quantitatively. A concrete mathematical background behind the generation of new resonant modes along with traditional $\mathrm{TM}_{10}$ mode is presented. An easy and handful formulation has been established for calculation of the frequency for new resonant mode as a function of antenna parameters and the gap between the septums. The detailed variation of resonant frequency of the new resonant mode as a function of the gap between the septums has been studied and presented in the paper. The proposed antenna can produce radiation along its broadside direction for two individual frequency band with single polarization. The theory presented in the paper is successfully utilized to evaluate the behavior of such antenna which is verified using [13] and close agreement is revealed.

The novelty of the proposed antenna is to excite a new resonant mode with radiation characteristics similar to that due to fundamental dominant $\mathrm{TM}_{10}$ mode which can be justified quantitatively with concrete mathematical background. Moreover, the antenna exhibit dual band operation with polarization purity in its radiation characteristics for both the bands and its structure is very simple and easy to manufacture.

The total work is analytically presented in the subsequent sections. First we have discussed the proposed antenna geometry in Section 2 followed by the results obtained from the new antenna at Section 3. In the next section (Section 4), thorough quantitative analysis in the background of transmission line model and Transverse

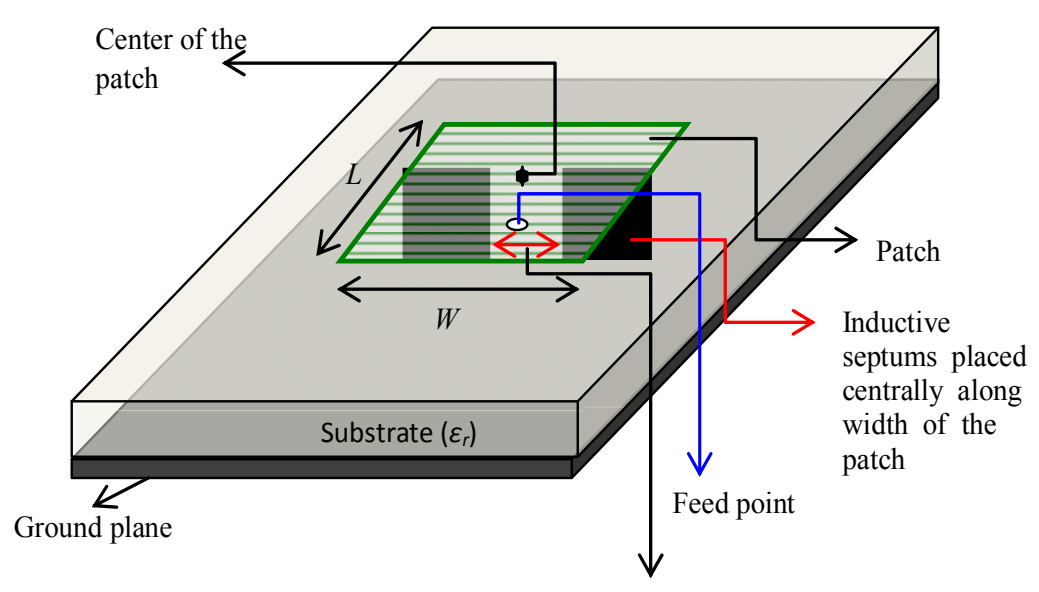

(a)
Gap ' $d$ ' between the septums

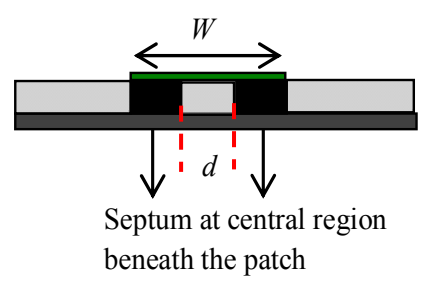

(b)

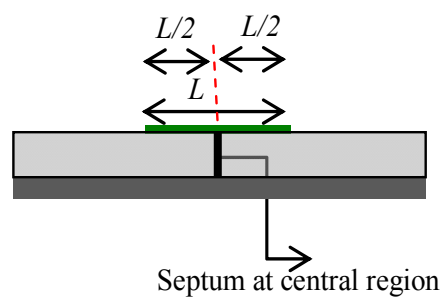

beneath the patch

(c)

Figure 1. Rectangular microstrip antenna with inductive septum placed beneath the patch centrally along the width of the patch. (a) overall antenna structure; (b) cross-sectional view along the width $(W)$; (c) longitudinal view/cross-sectional view along the length $(L)$. 
Resonance Method is presented to explain the new findings from the proposed antenna.

\section{Proposed Antenna Structure}

A rectangular microstrip antenna with length $L=12 \mathrm{~mm}$ and width $W=18 \mathrm{~mm}$ for $\mathrm{C}$ band operation is shown in Figure 1. A dielectric material of thickness $h=1.575$ $\mathrm{mm}$ and relative permittivity of $\varepsilon_{r}=2.33$ is utilized as substrate. A pair of simple thin metal plate (inductive septums) has been introduced beneath the patch centrally along the width of the patch as shown in the figure. The gap between the septums $d=6 \mathrm{~mm}$ is as shown.

\section{Results and Discussions}

The input and radiation characteristics for the proposed antenna operating in $\mathrm{C}$ band is presented with the help of FEM based software tool [14].

Figure 2 shows the complete return loss profile for both the conventional antenna and the proposed one (both having same $L, W$ and $h$ values) which indicates clearly that there is a generation of new mode along with the traditional $\mathrm{TM}_{10}$ mode in case of proposed antenna while this is not the case for conventional one. The conventional antenna resonates at $7.17 \mathrm{GHz}$ with an impedance bandwidth of $2.8 \%$, while the proposed antenna resonates at two individual frequency with $6.34 \mathrm{GHz}$ and $7.24 \mathrm{GHz}$ having $6 \%$ and $8 \%$ of impedance bandwidth respectively as revealed from Figure 2. Using the formulation developed by one of the present authors [15] it is seen that the patch with $L=12 \mathrm{~mm}, W=18 \mathrm{~mm}$ and $h=1.575 \mathrm{~mm}$ resonates at its fundamental dominant mode frequency of 7.2 GHz. Thus it confirms the development of new resonant mode at a frequency below the traditional fundamental $\mathrm{TM}_{10}$ mode for the proposed antenna. Figure 3 shows the radiation pattern for $\mathrm{E}$ and $\mathrm{H}$ plane of the proposed antenna for two separate frequencies. Figure 3(a) gives the radiation pattern at $7.24 \mathrm{GHz}$ i.e. the radiation fields associated with traditional $\mathrm{TM}_{10}$ mode where as 3(b) gives the radiation pattern at $6.33 \mathrm{GHz}$ i.e. the radiation fields associated with new mode. It may also be noted that for both the frequencies, radiations are in broad side direction as it should be for ideal dual band antennas. The gain for traditional $\mathrm{TM}_{10}$ mode is $7.2 \mathrm{dBi}$ while that for new mode is $6.2 \mathrm{dBi}$. The comparison of Figures 3(a) and (b) reveals that, the $\mathrm{H}$ plane cross polarized radiation associated with new resonant mode is slightly more than that of traditional $\mathrm{TM}_{10}$ mode while the co-polarized radiation pattern is nearly identical for both the frequencies. This slightly increased cross polarized radiation for the new mode does not hamper the antenna characteristic much but it may be minimized by cupping the ground plane as done in [16] for conventional microstrip antenna.

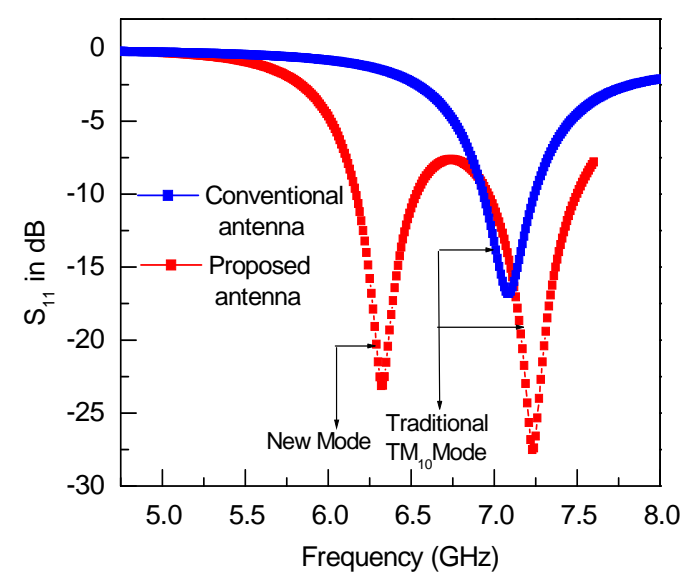

Figure 2. Complete return loss profile for conventional antenna and proposed structure. Parameters: $L=12 \mathrm{~mm}, W=$ $18 \mathrm{~mm}, h=1.575 \mathrm{~mm}, \varepsilon_{r}=2.33, d=6 \mathrm{~mm}$ and fed at $2.8 \mathrm{~mm}$ from center of the patch.

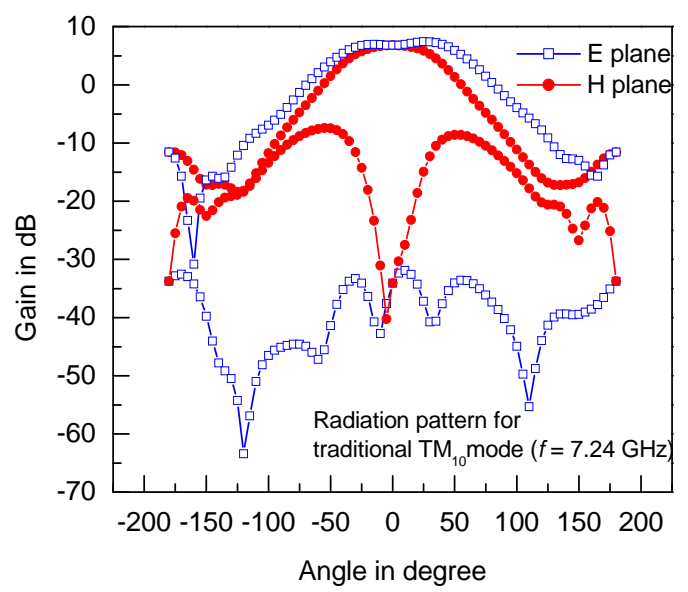

(a)

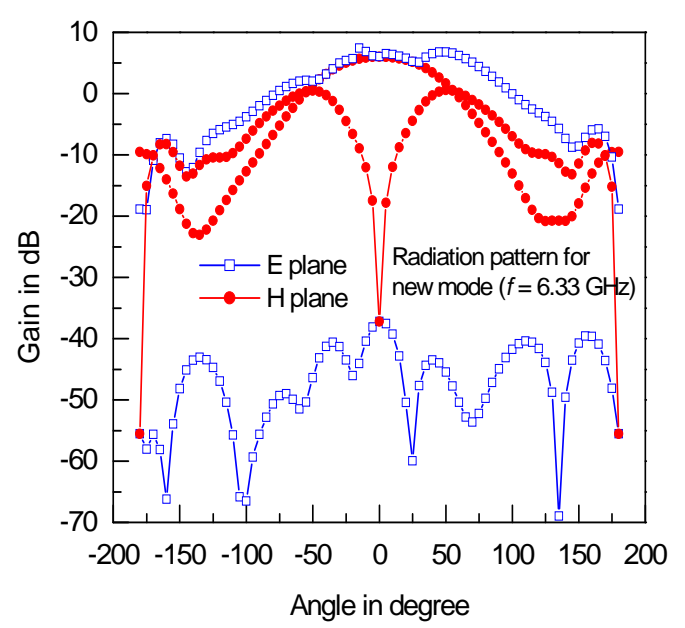

(b)

Figure 3. Radiation pattern for $E$ and $H$ planes of the proposed antenna. (a) at $7.24 \mathrm{GHz}$ i.e. the pattern associated with traditional $\mathrm{TM}_{10}$ mode; (b) at $6.33 \mathrm{GHz}$ i.e. the pattern associated with new mode. Parameters as in Figure 2. 
The effect of the separation between the septums $d$ on the resonant frequency of new mode in case of proposed antenna has been presented in Table 1. It is evident from the table that as the separation between the septums $d$ increases, resonant frequency of the new mode decreases. It should also be mentioned that, for other values of $d$ except $d=6 \mathrm{~mm}$, the matching is poor and hence the gain of the antenna at new mode is degraded than that for traditional $\mathrm{TM}_{10}$ mode. This is the reason of choosing $d=$ $6 \mathrm{~mm}$ for the proposed antenna.

\section{Quantitative Analysis}

When the very thin septums have been introduced underneath the patch along its width through central line (as in Figure 1), it produces an inductive impedance of $X_{L}$. As this discontinuity under the patch is placed along the central region, it will not perturb the field distribution for dominant $\mathrm{TM}_{10}$ mode. Because, for that dominant $\mathrm{TM}_{10}$ mode, the field distribution underneath the patch is such that there will be a nodal point of electric field at the central region. Now, the radiating slots can be assumed to be an open circuit point because we know that, these are considered as magnetic walls in cavity model. The equivalent transmission line model is as shown in Figure 4.

Thus the resultant impedance $\left(Z_{q}\right)$ transformed from these two radiating slots (open circuit points) at the central region will become parallel to the inductance produced by septum $X_{L}$ in that region and hence producing the equivalent impedance $Z_{E}$ at the central region below the patch.

If we consider that the patch and ground plane as parallel plate guide of characteristic impedance $Z_{0}$ and the impedance transformed from each radiating slot (open circuit points) at the central region is $Z_{s}$, then we may write

$$
Z_{s}=-j Z_{0} \cot \beta L / 2
$$

$$
Z_{q}=\frac{Z_{s}^{2}}{2 Z_{s}}=-j \frac{Z_{0}}{2} \cot \beta L / 2
$$

As, $Z_{E}$ is parallel combination of $Z_{q}$ and $j X_{L}$

$$
\begin{aligned}
& Z_{E}=\frac{Z_{q} \times j X_{L}}{\left[Z_{q}+j X_{L}\right]} \\
& Z_{E}=\frac{\left(-j \frac{Z_{0}}{2} \cot \beta L / 2\right) j X_{L}}{\left[-j \frac{Z_{0}}{2} \cot \beta L / 2+j X_{L}\right]}
\end{aligned}
$$

Following [17], if we apply Transverse Resonance Method (TRM) at the central region (where electric field node exists for $\mathrm{TM}_{10}$ mode) below the patch, we can write
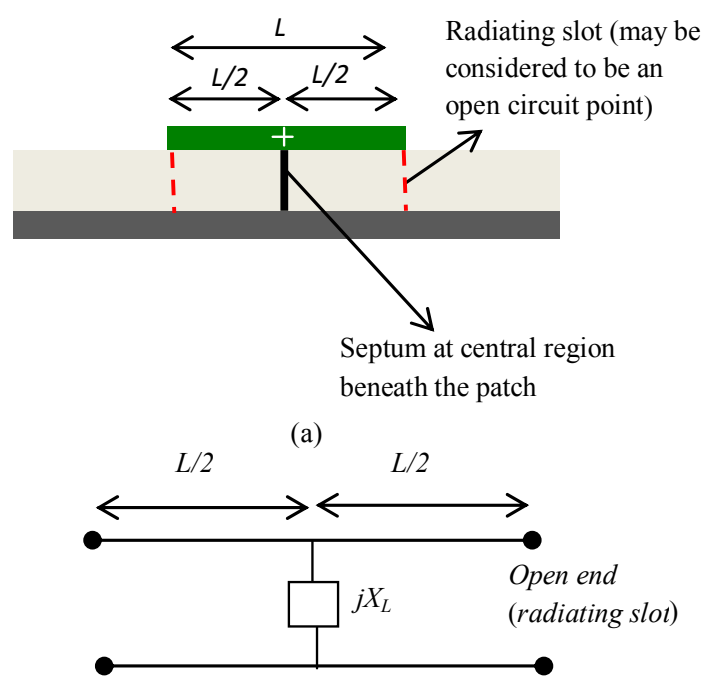

(b)

Figure 4. Cross-sectional view along the length $(L)$ for proposed structure and its equivalent transmission line model

\begin{tabular}{|c|c|c|c|c|}
\hline \multirow[t]{2}{*}{ Gap between septums $(d)$ in $\mathrm{mm}$} & \multicolumn{2}{|c|}{$\begin{array}{l}\text { Conventional microstrip antenna with: } L=12 \mathrm{~mm} \text {, } \\
W=18 \mathrm{~mm}, h=1.575 \mathrm{~mm}, \varepsilon_{r}=2.33 \text { and fed at } 2.8 \mathrm{~mm} \\
\text { from center of the patch }\end{array}$} & \multicolumn{2}{|c|}{$\begin{array}{l}\text { Proposed microstrip antenna with: } L=12 \mathrm{~mm}, \\
W=18 \mathrm{~mm}, h=1.575 \mathrm{~mm}, \varepsilon_{r}=2.33 \text { and fed at } \\
2.8 \mathrm{~mm} \text { from center of the patch; } \boldsymbol{d} \text { : variable }\end{array}$} \\
\hline & $f_{r}^{\mathrm{TM}_{10}} \mathrm{GHz}$ & $f_{r}^{\mathrm{New}} \mathrm{GHz}$ & $f_{r}^{\mathrm{TM}_{10}} \mathrm{GHz}$ & $f_{r}^{\mathrm{New}} \mathrm{GHz}$ \\
\hline 4.0 & & & 7.24 & 6.56 \\
\hline 4.6 & & & 7.22 & 6.50 \\
\hline 5.0 & 7.17 & No existence & 7.23 & 6.46 \\
\hline 6.0 & & & 7.24 & 6.34 \\
\hline 7.0 & & & 7.22 & 6.18 \\
\hline
\end{tabular}
(a) structural view; (b) transmission line equivalence.

Table 1. Variation of resonant frequency of new mode as a function of the gap between the septums $d$ for proposed antenna compared with conventional antenna. 


$$
\begin{aligned}
& Z_{E}=0 \\
& \Rightarrow \\
& \frac{\left(-j \frac{Z_{0}}{2} \cot \beta L / 2\right) j X_{L}}{\left[-j \frac{Z_{0}}{2} \cot \beta L / 2+j X_{L}\right]}=0
\end{aligned}
$$

This is possible if,

$$
\left(-j \frac{Z_{0}}{2} \cot \beta L / 2\right) j X_{L}=0
$$

Thus,

$$
\begin{aligned}
& \cot \beta L / 2=0=\cot \pi / 2 \\
& \Rightarrow \\
& L=\frac{\pi}{\beta}=\lambda_{g} / 2
\end{aligned}
$$

where $\lambda_{g}$ is the wavelength within the dielectric.

Which is the primary requirement for the existence of dominant $\mathrm{TM}_{10}$ mode underneath the patch. Thus, it further confirms that the inductive septum placed underneath the patch along its width at the central region which produces an inductive impedance of $X_{L}$ will not affect the field distribution of dominant $\mathrm{TM}_{10}$ mode and hence radiation associated with this mode is kept unaltered.

Now we may concentrate on the effect of inductive septum to produce the new resonant mode below the traditional $\mathrm{TM}_{10}$ mode resonance. In fact, the effect becomes prominent at a frequency other than $\mathrm{TM}_{10}$ mode frequency. In that case, the nodal point of electric field does not exist at the central region where septums are introduced. If we consider only one side of length $L / 2$ (i.e. from the central region towards one radiating slot) (see Figure 4), at the central region beneath the patch, the equivalent impedance due to one side $Z_{E}^{1}$ as

$$
Z_{E}^{1}=\frac{Z_{s} \times j X_{L}}{\left[Z_{s}+j X_{L}\right]}
$$

as the impedance transformed from each radiating slot (open circuit points) at the central region is $Z_{s}$. This is basically the equivalent impedance at the central region below the patch due to parallel combinations of impedance transformed from one radiating slot (open circuit point) (as we have considered only one side) and the inductive impedance generated due to introduction of septum at the central region.

Then using Equations (1) and (9)

$$
Z_{E}^{1}=\frac{\left(-j Z_{0} \cot \beta L / 2\right) j X_{L}}{\left[-j Z_{0} \cot \beta L / 2+j X_{L}\right]}
$$

$$
\begin{gathered}
=\frac{\left(Z_{0} \cot \beta L / 2\right) X_{L}}{j\left[X_{L}-Z_{0} \cot \beta L / 2\right]} \\
Z_{E}^{1}=\frac{-j\left(Z_{0} \cot \beta L / 2\right) X_{L}}{\left[X_{L}-Z_{0} \cot \beta L / 2\right]}
\end{gathered}
$$

which in fact, is capacitive in nature as observed from Equation (11). Now, if we consider this capacitance is developed at the input of an open circuited transmission line of length $\Delta l$, then we may write

$$
-j Z_{0} \cot \beta \Delta l=Z_{E}^{1}=\frac{-j\left(Z_{0} \cot \beta L / 2\right) X_{L}}{\left[X_{L}-Z_{0} \cot \beta L / 2\right]}
$$

Thus the effective position of the open circuit point is then located outward at a distance of $\Delta l$ from the central region. Now using [18,19]

$$
X_{L}=\left(\frac{W}{\lambda_{g}}\right) Z_{0} \tan ^{2}\left(\frac{\pi d}{2 W}\right)
$$

Now with $d=6 \mathrm{~mm}, W=18 \mathrm{~mm}$ we have

$$
X_{L}=Z_{0}\left(\frac{6}{\lambda_{g}}\right)
$$

Thus from Equations (12)-(14), we can write

$$
\begin{aligned}
& -j Z_{0} \cot \beta \Delta l=\frac{-j\left(Z_{0} \cot \beta L / 2\right) Z_{0}\left(\frac{6}{\lambda_{g}}\right)}{\left[\left(\frac{6}{\lambda_{g}}\right) Z_{0}-Z_{0} \cot \beta L / 2\right]} \\
& \cot \beta \Delta l=\frac{(\cot \beta L / 2)\left(\frac{6}{\lambda_{g}}\right)}{\left[\left(\frac{6}{\lambda_{g}}\right)-\cot \beta L / 2\right]} \\
& \left(\frac{6}{\lambda_{g}}\right) \cot \beta \Delta l-\cot \beta \Delta l \cot \beta L / 2 \\
& =\left(\frac{6}{\lambda_{g}}\right) \cot \beta L / 2
\end{aligned}
$$

Let,

$$
\begin{aligned}
& \cot \beta L / 2=m \\
& \cot \beta \Delta l=n
\end{aligned}
$$

Thus using (17)-(19)

$$
\left(\frac{6}{\lambda_{g}}\right) n-m n=\left(\frac{6}{\lambda_{g}}\right) m
$$

dividing both the sides by $m n$ 


$$
\begin{aligned}
& {\left[\frac{6 / \lambda_{g}}{m}\right]-1=\left[\frac{6 / \lambda_{g}}{n}\right]} \\
& {\left[\frac{6 / \lambda_{g}}{m}\right]-\left[\frac{6 / \lambda_{g}}{n}\right]=1}
\end{aligned}
$$

Hence,

Thus

$$
\begin{aligned}
& {\left[\frac{6 / \lambda_{g}}{m}\right] \triangleright\left[\frac{6 / \lambda_{g}}{n}\right] \Rightarrow m \triangleleft n} \\
& \Rightarrow \cot \beta L / 2 \triangleleft \cot \beta \Delta l
\end{aligned}
$$

$$
\beta L / 2 \triangleleft \beta \Delta l \Rightarrow \Delta l \triangleright L / 2
$$

Thus the effective position of the open circuit point (which may be assumed to be one radiating slot) is then located outward at a distance of $\Delta l$ from the central region and this is greater than the half of the length of the patch. Thus, the actual radiating slot in one side is at a distance of $L / 2$ from the central region while another effective radiating slot is developed and the effective position of that new radiating slot in one side is at a distance of $\Delta l$ from the central region. The equivalent transmission line model for both the traditional $\mathrm{TM}_{10}$ mode and the new mode is as shown in Figure 5.

Thus, considering both the sides, these two effective radiating slots are separated by $2 \Delta l$. This length is acting as a resonant length for a new resonant mode and thus a new mode has been generated. This new resonant mode radiates the electromagnetic wave through these effective radiating slots. However, the conventional dominant resonant mode $\mathrm{TM}_{10}$ kept unaltered as introduction of inductive septum does not affect the field distribution as explained earlier.

Now, from Equation (24)

$$
2 \Delta l \triangleright L
$$

Let, the resonant frequency of conventional dominant
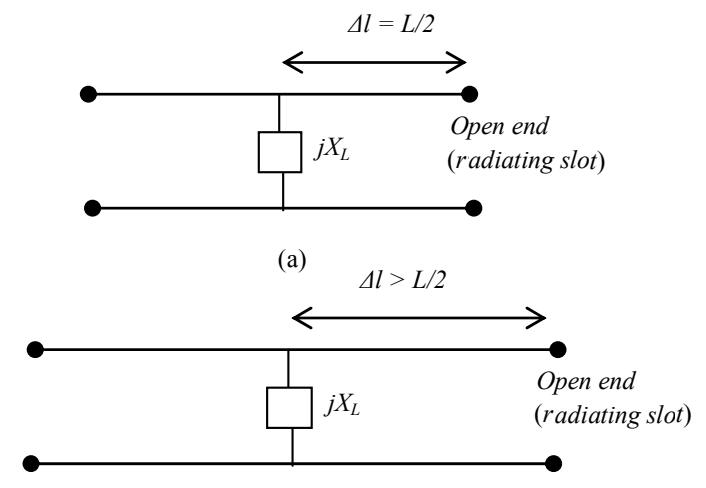

(b)

Figure 5. Equivalent transmission line model for traditional $\mathrm{TM}_{10}$ mode and new mode (a) traditional $\mathrm{TM}_{10}$ mode; (b) new mode. resonant mode $\mathrm{TM}_{10}$ is $f_{r}^{\mathrm{TM}_{10}}$ and that for new mode $f_{r}^{\text {New }}$. Thus,

$$
\begin{aligned}
& f_{r}^{\mathrm{TM}_{10}}=c / 2 L \sqrt{\varepsilon_{r}} \\
& f_{r}^{\mathrm{New}}=c / 2(2 \Delta l) \sqrt{\varepsilon_{r}}
\end{aligned}
$$

Using Equation (25)

$$
f_{r}^{\mathrm{New}} \triangleleft f_{r}^{\mathrm{TM}_{10}}
$$

Thus the new mode with a resonant frequency lesser than that for traditional dominant $\mathrm{TM}_{10}$ mode is generated with the proposed antenna.

Now, if the gap between the septums $d$ increases, the resonant frequency of the new mode decreases. Let us consider two cases, where the gap between septums are $d_{1}$ and $d_{2}$. The inductive reactances for those are $X_{L 1}$ and $X_{L 2}$ respectively.

Let, $d_{1} \triangleright d_{2}$

Thus from Equation (13),

$$
\tan ^{2} \pi d_{1} / 2 W \triangleright \tan ^{2} \pi d_{2} / 2 W \Rightarrow X_{L 1} \triangleright X_{L 2}
$$

where in the proposed structure, the gap between the septums is limited.

Thus,

$$
\begin{gathered}
0 \triangleleft d_{1} \triangleleft W \\
\pi / 2 \triangleright \pi d_{1} / 2 W \triangleright 0
\end{gathered}
$$

similarly;

$$
\begin{gathered}
0 \triangleleft d_{2} \triangleleft W \\
\pi / 2 \triangleright \pi d_{2} / 2 W \triangleright 0
\end{gathered}
$$

Thus recalling Equation (12) for these two cases,

$$
\cot \beta \Delta l_{1}=\frac{\left(X_{L 1} \times \cot \beta L / 2\right)}{\left[X_{L 1}-Z_{0} \cot \beta L / 2\right]}
$$

and

$$
\cot \beta \Delta l_{2}=\frac{\left(X_{L 2} \times \cot \beta L / 2\right)}{\left[X_{L 2}-Z_{0} \cot \beta L / 2\right]}
$$

dividing numerator and denominator of right hand side of Equations (30) and (31) by $X_{L 1}$ and $X_{L 2}$ respectively,

$$
\begin{aligned}
& \cot \beta \Delta l_{1}=\frac{(\cot \beta L / 2)}{\left[1-\frac{Z_{0} \cot \beta L / 2}{X_{L 1}}\right]} \\
& \cot \beta \Delta l_{2}=\frac{(\cot \beta L / 2)}{\left[1-\frac{Z_{0} \cot \beta L / 2}{X_{L 2}}\right]}
\end{aligned}
$$

using (29)

$$
\cot \beta \Delta l_{1} \triangleleft \cot \beta \Delta l_{2}
$$

hence, 


$$
\Delta l_{1} \triangleleft \Delta l_{2}
$$

Thus the two new effective radiating slots are separated by $2 \Delta l_{1}$ which is smaller when the gap between septums are $d_{1}$, compared to that when the gap between septums are $d_{2}$. This confirms the observation of Table 1, which show that, as the gap between septums increase, the resonant frequency of new resonant mode decrease.

Now in case of proposed antenna of $L=12 \mathrm{~mm}, W=$ $18 \mathrm{~mm}$ and the gap between the septum is $d=6 \mathrm{~mm}$, Equations (18) and (19) gives,

$$
m=\cot \beta L / 2=\cot 12 \pi / \lambda_{g}
$$

using Equations (16), (19) and (36),

$$
n=\cot \beta \Delta l=\frac{(\cot \beta L / 2)\left(\frac{6}{\lambda_{g}}\right)}{\left[\left(\frac{6}{\lambda_{g}}\right)-\cot \beta L / 2\right]}
$$

Thus,

$$
n=\frac{\left(\cot 12 \pi / \lambda_{g}\right)\left(\frac{6}{\lambda_{g}}\right)}{\left[\left(\frac{6}{\lambda_{g}}\right)-\cot 12 \pi / \lambda_{g}\right]}
$$

Using the formulation developed in (21),

$$
\left[\frac{6 / \lambda_{g}}{m}\right]-\left[\frac{6 / \lambda_{g}}{n}\right]=1
$$

now, putting the values from Equations (36) and (37) above equation results

$$
\left[\frac{\left(6 / \lambda_{g}\right)}{\cot 12 \pi / \lambda_{g}}\right]-\left[\frac{\left(6 / \lambda_{g}\right)\left[\left(\frac{6}{\lambda_{g}}\right)-\cot 12 \pi / \lambda_{g}\right]}{\left(\cot 12 \pi / \lambda_{g}\right)\left(\frac{6}{\lambda_{g}}\right)}\right]=1
$$

which can be solved by numerical technique or graphical method and found to be satisfied for $\lambda_{g}=31.048 \mathrm{~mm}$.

Thus, $\lambda=\lambda_{g} \sqrt{\varepsilon_{r}}=47.39 \mathrm{~mm}$ and $\left(f_{r}^{\text {New }}\right)=c / \lambda=6.33 \mathrm{GHz}$ for $d=6 \mathrm{~mm}$ which is in excellent agreement with the simulated results as revealed from Figure 2 and Table 1.

\section{Conclusion}

A rectangular microstrip antenna with centrally loaded inductive septums below the patch is proposed for dual band operation. The superiority of the proposed antenna to excite a new resonant mode below the conventional $\mathrm{TM}_{10}$ mode with faithful radiation pattern and appreciable impedance bandwidth for both the bands is verified along with concrete mathematical justification. A clearcut and handful relationship has been established and successfully utilized for calculation of the frequency for new resonant mode for proposed antenna. This will be unquestionably helpful for the scientists, researchers and practicing engineers looking for such low profile antenna with dual band operation. The thorough mathematical analysis for the generation of new mode using such antenna is very supportive for future investigations in this area.

\section{REFERENCES}

[1] R. Garg, P. Bhartia, I. Bahl and A. Ittipiboon, "Microstrip Antenna Design Handbook," Artech House, Norwood, 2001.

[2] S. Maci and G. B. Gentili, "Dual-Frequency Patch Antennas," IEEE Antennas and Propagation Magazine, Vol. 39, No. 6, 1997, pp. 13-20. doi:10.1109/74.646798

[3] J.-S. Chen and K.-L. Wong, "A Single-Layer Dual-Frequency Rectangular Microstrip Patch Antenna Using a Single Probe Feed," Microwave and Optical Technology Letters, Vol. 11, No. 2, 1996, pp. 38-84. doi:10.1002/(SICI)1098-2760(19960205)11:2<83::AIDMOP10>3.0.CO;2-8

[4] Y. M. M. Antar, A. I. Ittipiboon and A. K. Bhattachatyya, "A Dual-Frequency Antenna Using a Single Patch and An Inclined Slot," Microwave and Optical Technology Letters, Vol. 8, No. 6, 1995, pp. 309-310. doi:10.1002/mop.4650080613

[5] J. S. Dahele, K. F. Lee and D. P. Wong, "Dual Frequency Stacked Annular-Ring Microstrip Antenna," IEEE Transactions on Antennas and Propagation, Vol. 35, No. 11, 1987, pp. 1281-1285. doi:10.1109/TAP.1987.1143997

[6] J. Wang, R. Fralich, C. Wu and J. Litva, "Multifunctional Aperture Coupled Stack Antenna," Electronics Letters, Vol. 26, No. 25, 1990, pp. 2067-2068. doi:10.1049/el:19901333

[7] W. F. Richards, S. E. Davidson and S. A. Long, "DualBand Reactively Loaded Microstrip Antenna," IEEE Transactions on Antennas and Propagation, Vol. 33, No. 5, 1985, pp. 556-560. doi:10.1109/TAP.1985.1143617

[8] S. E. Davidson, S. A. Long and W. F. Richards, "DualBand Microstrip Antenna with Monolithic Reactive Loading," Electronics Letters, Vol. 21, No. 21, 1985, pp. 936 937. doi:10.1049/el:19850662

[9] H. Nakano and K. Vichien, "Dual-Frequency Patch Antenna with a Rectangular Notch," Electronics Letters, Vol. 25, No. 16, 1989, pp. 1067-1068. doi:10.1049/el:19890714

[10] J.-H. Lu and K.-L. Wong, "Slot-Loaded, Meandered Rectangular Microstrip Antenna with Compact Dual Frequency Operation," Electronics Letters, Vol. 34, No. 11, 1998, pp. 1048-1050. doi:10.1049/el:19980737

[11] K.-L. Wong and K.-P. Yang, "Small Dual-Frequency Microstrip Antenna with Cross Slot," Electronics Letters, Vol. 33, No. 23, 1997, pp. 1916-1917. 
doi:10.1049/el:19971355

[12] K.-L. Wong and W.-S. Chen, "Compact Microstrip Antenna with Dual-Frequency Operation," Electronics Letters, Vol. 33, No. 8, 1997, pp. 646-647. doi:10.1049/el:19970433

[13] X. L. Bao and M. J. Ammann, "Dual-Frequency Circularly-Polarized Patch Antenna with Compact Size and Small Frequency Ratio," IEEE Transactions on Antennas and Propagation, Vol. 55, No. 7, 2007, pp. 2104-2107. doi:10.1109/TAP.2007.900271

[14] "High Frequency Structure Simulator," Ansoft Corp, Version 10.

[15] S. Chattopadhyay, M. Biswas, J. Y. Siddiqui and D. Guha, "Rectangular Microstrips with Variable Air-Gap Varying Aspect Ratio: Improved Formulation and Experiments," Microwave Optical Technology Letters, Vol. 51, No. 1, 2009, pp. 169-173. doi:10.1002/mop.24025
[16] K. Malakar, J. Nandi, S. Mitra, P. K. Gorai, S. Chattopadhyay, J. K. Sah and A. Anand, "Physical Insight into the Low Cross Polarized Radiation with Rectangular Microstrip Antenna on Cupped Ground Plane," International Journal of Electrical, Electronics and Computer Systems, Vol. 6, No. 2, 2012, pp. 1-5.

[17] S. K. Ghosh, A. Ghosh, D. Ghosh, S. Chattopadhyay and S. Banerjee, "Rectangular Microstrip Antenna on Ridge Ground Plane to Control the Resonant Modes for Improved Bandwidth using Transverse Resonance Method," Journal of Electromagnetic Analysis and Applications, Vol. 4, No. 5, 2012, pp. 206-211. doi:10.4236/jemaa.2012.45028

[18] P. A. Rizzi, "Microwave Engineering: Passive Circuits," Prentice Hall, Upper Saddle River, 1999.

[19] R. E. Collin, "Foundation for Microwave Engineering," 2nd Edition, IEEE Press, Hoboken, 2003. 